\title{
CLIENT-SERVER SYSTEM MOBILE EXPERIMENT CONTROL AND DATA ANALYSIS WITH OBLIQUE IONOSPHERIC SOUNDING
}

\author{
Vladimir Ivanov* \\ Volga State University of Technology, Yoshkar-Ola, Russia \\ Dmitriy Ivanov \\ Volga State University of Technology, Yoshkar-Ola, Russia \\ Natalya Ryabova \\ Volga State University of Technology, Yoshkar-Ola, Russia \\ Andrei Chernov \\ Volga State University of Technology, Yoshkar-Ola, Russia \\ Michael Shalagin \\ Volga State University of Technology, Yoshkar-Ola, Russia
}

We propose an approach to solving the problem of mobile management and the analysis of data of oblique sounding of an ionosphere and ionospheric HF radio channels on a network of radio links. On the basis of our research, we have shown the way to solve this problem, illustrated by diagrams of client-server architectures, mobile client interface and a mobile system to interaction with the receiving terminal.

Key words: Client-server system, Oblique ionospheric sounding, Architecture, Mobile experiment control

\section{INTRODUCTION}

At present, a wide spread of mobile devices opens up new opportunities created by mobile applications. An important direction of this process is the use of mobile applications in scientific experimental studies [03, 04]. In addition, advances in computer technology led to the development direction for the creation of universal radio systems, the basic functionality that is implemented in software (SDR). Created a network of automated HF communications for organizations that require adaptive, small radiosonde system ionospheric radio transmitting and receiving terminals such systems may be separated by thousands of kilometers. The Volga State University of Technology (VSUT) developing scientific direction connected to remote sounding of the ionosphere and ionospheric HF communication channel using complex decameter radio signals. Therefore, the actual scientific task is to study the possibility of remote control of parameters of network terminals of sensing and analysis of experimental data obtained in points distant from them over long distances, using mo- bile devices and information technologies. This is important when the terminals allow software to reconstruct their function realizing how the communication system and sounding system of ionospheric radio link. Purpose - to develop a system for remote mobile experiment control and data analysis with oblique ionospheric sounding chirp signal with using of network technologies.

\section{MONITORING SYSTEM OF HF RADIOCHANNELS}

Specialities research is radio-sounding ionosphere on HF. The applied nature of the work associated with the use of the results of sensing to improve the efficiency of long-distance (thousands of kilometers) HF communications and radar horizon. Radiotechnical systems (RTS) of radar and communications in general, have the structure shown in Figure 1 [10, 02]. Communication between the transmitting and receiving terminals is carried out by the presence of the communication line in a physical environment - ionosphere in which radio signals spread in the form of radio waves. It is assumed that the radio is part of the RTS. 


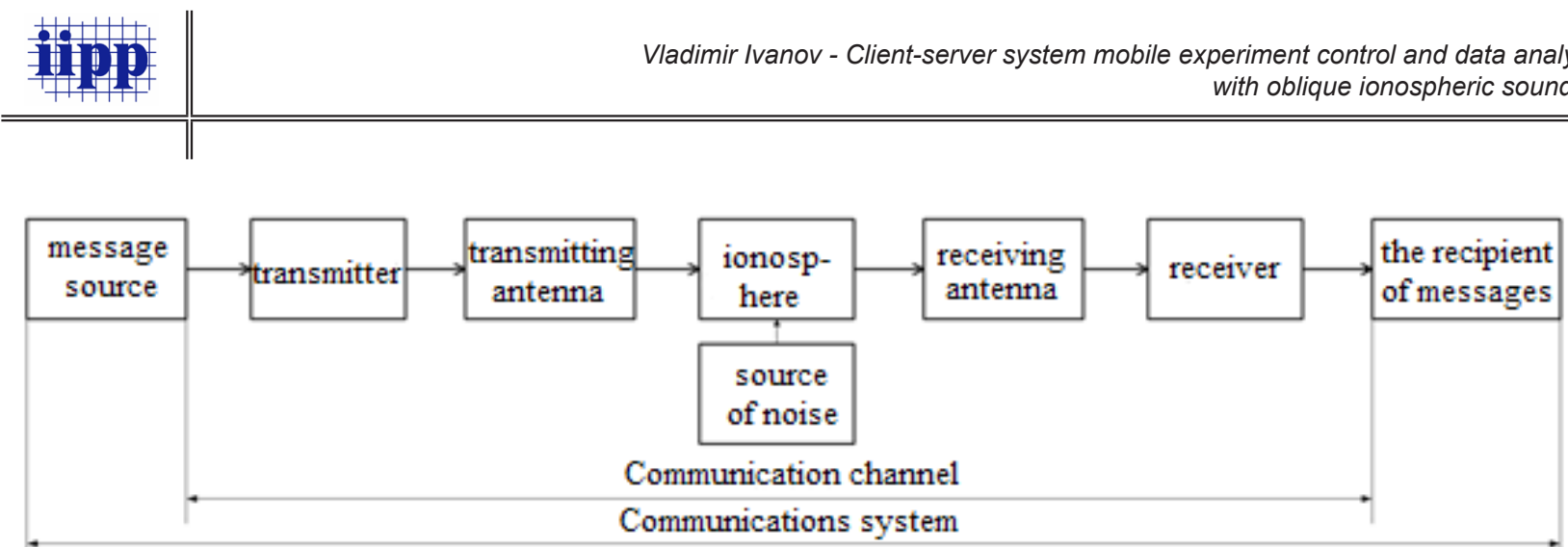

Figure 1: The general structure of the RTS, used for communicate the physical environment - the ionosphere

Typically, signals are determined by several parameters, information on which is important in processing signals in receiver to isolate them useful information. These parameters define the information - technical specifications (ITS) of RTS. However, the characteristics of the radio channel can be matched to the signal, resulting in distortion of signals in the radio channel and adversely affect the operation of the RTS. The approval procedure ITS of RTS channel parameters must be adaptive. This is due to the fact that the channel parameters are experiencing constant change, caused by changes in the characteristics of the ionosphere of the Earth's rotation around its axis (daily) and around the sun (seasonal), as well as due to changes in solar activity, and others. The variations have different scales (periods - in time domain and frequency - in frequency domain).

To solve the problem of adaptation is used ionosphere sounding in the HF range, the results of which are evaluated parameters of frequency channels with different center frequencies and is determined by the range of permissible values for these frequencies from the lowest usable frequency (LUF) to the maximum usable frequency (MUF). The key parameters of the partial channels are: signal to noise ratio, scattering in the channel delay, scattering in the channel from the Doppler shift of the frequency [08, 07].

In 2013, the VSUT developed modernized version of lonosonde continuous chirp signal - on the SDR-platform USRP N210 with child boards and LFRX LFTX [8]. Experiments using a network of oblique radio-sounding ionosphere on long radio paths. This analysis of the experimental data is carried out in this case in the center of a network of Yoshkar-Ola.

Figure 2 is a simplified scheme of a mobile system to the receiving terminal.

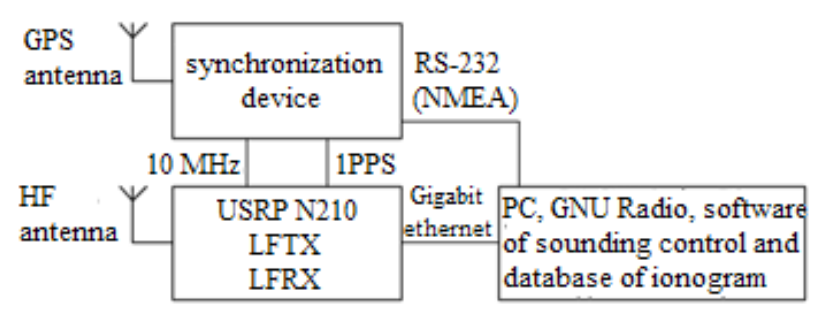

Figure 2: Scheme of the receiving terminal hardware and software LFM lonosonde

In the process, there was scientific and technical management problem lonosonde network and experimental data analysis parameters of the partial mobile radio from a remote point, request a relevant studies. Taken into account that develops mobile system should provide interaction with the device software defined lonosonde. Therefore, in the beginning it was planned to use remote administration tools (RD), such as TeamViewer, UltraVNC, FreeRDP etc. It turned out that they have significant disadvantages for mobile use:

- high requirements for the quality of the network link from the need to relay the contents of the screen through a computer network;

- slow response to user actions;

- lack of opportunity for simultaneous multi-clients;

- client has full control over the system, may inadvertently or intentionally disrupt the system.

\section{ARCHITECTURE \& UI}

We have proposed an approach that allows solve the scientific and technical problems by creating their own mobile client-server applications. It directly controls the backend process (in this case, ionosonde), and the client provides the user with the necessary interface and sends requests to perform management tasks and analysis. This solves most of the problems of RD-method but it's time consuming. 


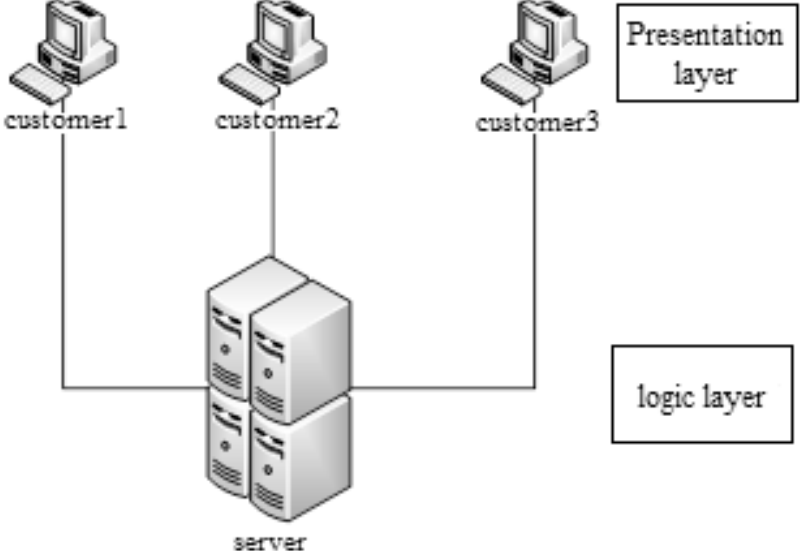

(a)

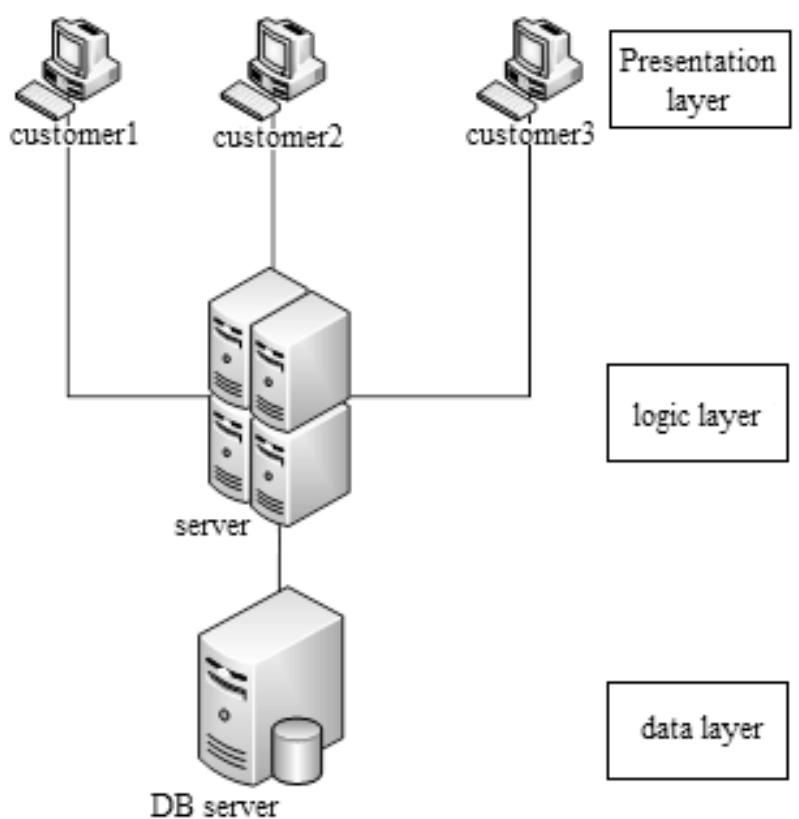

(b)

Figure 3: Scheme of client-server architectures, (a) - a two-level architecture, (b) - three-level architecture

It was considered that at the moment there are two major client-server applications - two-level and three-level [05], presented in Figure 3. In a two-level system architecture is divided into the presentation layer (user interface) and the level of logic (implementation of the basic functionality of the application). If a portion of logic transferred to the client, we speak of "thick" client, otherwise, the client is "thin". Three-level architecture distinguishes individual level data. At this level, the database server, usually represented by another computer. On the database server stores data, which are processed at the level of logic.

We have selected architecture "two-level clientserver Thin Client".

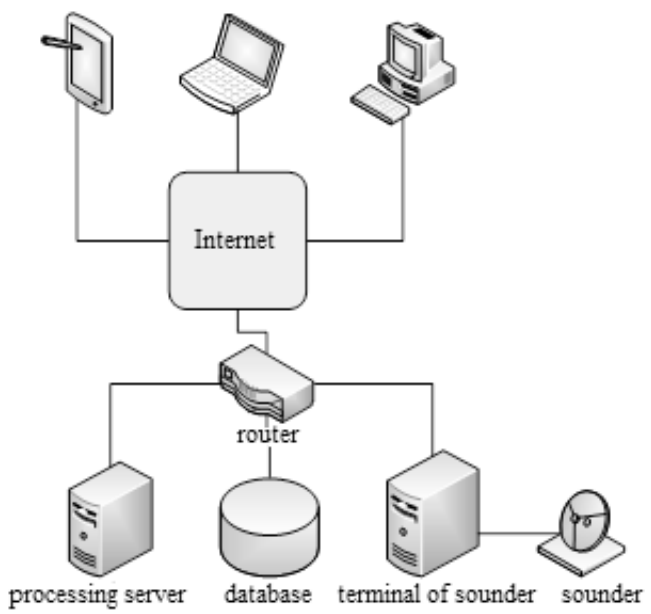

Figure 4: Scheme of the architecture of a mobile system to communicate with the receiving terminals
The scheme of this architecture is shown in Figure 4 and comprises:

- PC with GNU Radio to communicate with the USRP, and the management of the program Ionosonde [10], to receive commands from a server treatments;

- server treatments, which is a separate PC application that handles customer inquiries, performing secondary processing ionogram, transmitting the compressed file ionogram, daily moves and other results of processing a remote client, and sends commands to the control program lonosonde terminal. Also, the server is running a local NTP-server stores the cached results of previous treatments;

- network storage system (NAS), in which files are stored ionogram;

- clients - at the moment for OS Android.

Ionosonde terminal, server applications and the data warehouse connected to a local network via a router (you can use switches of 3 level). The router is configured so that access from the outside is possible only on certain ports server treatments. Server treatments, Ionosonde terminal and interact with customers we have implemented at the application layer protocol stack TCP / IP, based on the TCP. The client has three main scenarios for interaction with the system control parameters, lonosonde request and the request is received ionogram treatments. 
When prompted management ionosonde first checks whether the user has the privilege to manage ionosonde, and if the parameters are correct, the command is transmitted to the terminal lonosonde. When prompted ionogram verify whether it is in stock. If ionogram detected, it is transmitted to the client, otherwise the client is transferred to an error message.

When requesting treatments first checks the cache processing. If the treatment is found, it is sent to the client. If not, the server holds a secondary processing ionogram, transmits the result to the client and stores it in a cache.

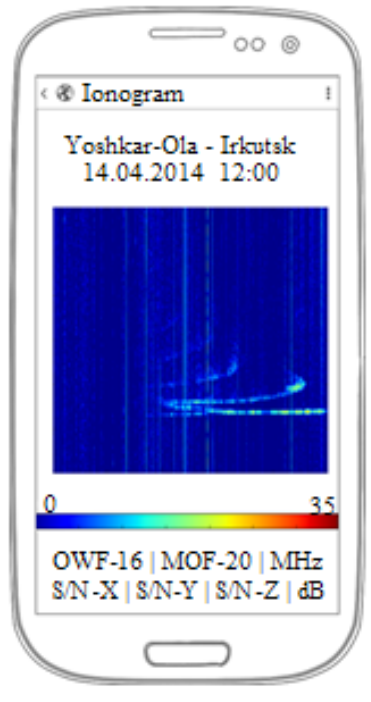

a)

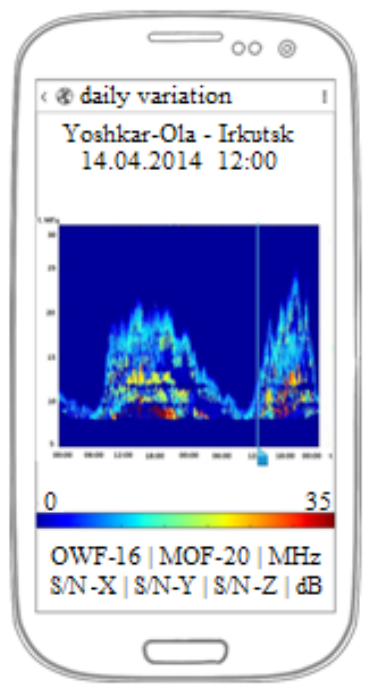

b)
Figure 5: Models of the mobile client interface, a) ionogram oblique sounding b) diurnal variation of energy of the impulse response of the channel

Terminal lonosonde copies obtained ionogram in the data store and adds a new entry to the database server ionogram treatments.

Mock-ups of mobile client interface shown in Figure 5. Here, a) - display the requested ionogram with the basic parameters of the channel, b) - shows the diurnal variation of the profile of the total energy of the impulse response with the main parameters of the channel for a given point in time.

Library networking is implemented in the programming language Java. Its main features are: cross-platform (OS Android, Windows, Linux, Mac OS X); custom communication protocol; asynchronous reception, transmission and processing of messages; link encryption algorithms for AES, DES and RSA.

\section{CONCLUSION}

We propose an approach to solving the problem of mobile management and the analysis of data of oblique sounding of an ionosphere and ionospheric HF radio channels on a network of radio links. Showing how to implement client-server architecture of a mobile system to communicate with the receiving terminals, the interface of the mobile client.

This work was supported by grants RFBR project № 13-07-00371; 13-02-00524; 15-07-05280; 15-0705294; Grants Ministry of Education of the Russian Federation № 3.2695.2014 / K, № 8.2697.2014 / K, № 2276, № 2247; RNF № 15-19-10053.

\section{REFERENCES}

1) $\mathrm{ACl}$ Mobile Banking http://www.aciworldwide. com/products-and-services/community-financial/ self-service-banking/aci-mobile-banking.aspx

2) А. В. Петров, О. С. Большаков, А. С. Лебедев, Н. Е. Голубева, Метод шаблонов приложений для повышения мобильности распределенных систем сбора и ретрансляции информации с биомедицинских датчиков, журнал радиоэлектроники, n5, 2013, удк 002.6:022

3) Fink, L.M.: Theory of transmission of discrete messages. Soviet Radio, (1970).

4) Google - Global Business Map http://ssl.gstatic. com/think/docs/global-business-map_researchstudies.pdf

5) http://www.hpc-education.ru/files/lectures/radchenko/radchenko_txt03.pdf

6) Ivanov, D.V., Ivanov, V.A., Ryabova, N.V., Elsukov, A.A., Ryabova, M.I., Chernov, A.A.: SDR-ionosonde with continuous LFM-signal on the platform USRP. Vestnik of Volga State University of Technology. Series "Radio Engineering and Infocommunication Systems", Vol. 19, No. 3, 80-93. (2013)

7) Ivanov, V.A., Ivanov, D.V., Ryabova, N.V., Chernov, A.A.: Multivariate high radio and experimental studies of its key features. Electromagnetic waves and electronic systems, Vol. 18, No. 8. 40-48. (2013)

8) Kryukovsky, A.S., Bunnies, I.V.: Features of distribution of radio pulses in media with dispersion. Electromagnetic waves and electronic systems, Vol. 13, No. 8, 36-41. (2008)

9) Maslin, N.M.: HF communications: a systems approach. London.: Pitman Publishing. (1987)

10) Soured, J.: Digital communication: lane. from English. Radio and Communications, (2000)

Paper sent to revision: 18.08.2015.

Paper ready for publication: 15.12.2015. 